\title{
Radiative Kähler moduli stabilization
}

\author{
Tatsuo Kobayashi, ${ }^{1, *}$ Naoya Omoto, ${ }^{1, \dagger}$ Hajime Otsuka, ${ }^{2, \$}$ and Takuya H. Tatsuishi ${ }^{1, \S}$ \\ ${ }^{1}$ Department of Physics, Hokkaido University, Sapporo 060-0810, Japan \\ ${ }^{2}$ Department of Physics, Waseda University, Tokyo 169-8555, Japan
}

(Received 19 January 2018; published 11 May 2018)

\begin{abstract}
We propose a new type of Kähler moduli stabilization mechanisms in type IIB superstring theory on Calabi-Yau manifolds with the positive Euler number. The overall Kähler modulus can be perturbatively stabilized by radiative corrections due to sparticles. Its minimum is the anti-de Sitter vacuum, where supersymmetry is broken. We can uplift it to the de Sitter vacuum by introducing anti-D-branes, keeping the modulus stabilized. Although our numerical results depend on the choice of the cutoff scale and degeneracies of sparticles, at any rate there exist the parameter spaces where the masses of Kaluza-Klein and stringy modes are larger than the cutoff scale. Furthermore, this stabilization scenario predicts an ultralight axion.
\end{abstract}

DOI: $10.1103 /$ PhysRevD.97.106006

\section{INTRODUCTION}

The moduli fields appear ubiquitously in four-dimensional low-energy effective field theory derived from superstring theory on six-dimensional compact space. These fields correspond to a geometrical character of compact space. Unless these moduli fields are stabilized at a high scale, it will lead to the fifth force. Hence, moduli stabilization is one of the major topics in the string cosmology and phenomenology.

So far, moduli stabilization has been well studied in the type IIB superstring theory on Calabi-Yau (CY) manifolds. The closed string moduli are categorized into the dilaton $S$, complex structure moduli $U$ and Kähler moduli $T .{ }^{1}$ Here, we denote the numbers of complex structure moduli and Kähler moduli by $h^{2,1}$ and $h^{1,1}$, which correspond to the numbers of three-cycles and four-cycles of CY, respectively. Three-form fluxes in the type IIB superstring theory can stabilize the dilaton $S$ and all the complex structure moduli $U$ at the compactification scale [3] and they generically generate the nonvanishing flux-induced superpotential $W_{0}=\left\langle W_{\text {flux }}\right\rangle$ [4]. The remaining Kähler moduli are stabilized at an anti-de Sitter minimum in the

\footnotetext{
*kobayashi@particle.sci.hokudai.ac.jp

tomoto@particle.sci.hokudai.ac.jp

th.otsuka@aoni.waseda.jp

\$th-tatsuishi@particle.sci.hokudai.ac.jp

${ }^{1}$ In this paper, we do not discuss the stabilization of open string moduli. For discussion of open string moduli in F-theory context, see, e.g., Ref. [1,2], where the open string moduli are identified with the complex structure moduli of CY fourfold.

Published by the American Physical Society under the terms of the Creative Commons Attribution 4.0 International license. Further distribution of this work must maintain attribution to the author(s) and the published article's title, journal citation, and DOI. Funded by SCOAP ${ }^{3}$.
}

Kachru-Kallosh-Linde-Trivedi (KKLT) scenario [5] and large volume scenario (LVS) [6] using the nonperturbative superpotential for the Kähler moduli. Such an anti-de Sitter minimum is uplifted to a metastable de Sitter minimum by introducing anti-D3 branes [5]. The $F$-term uplifting scenario is another way to realize the de Sitter minimum $[7,8]$. In the KKLT scenario, the stabilization of Kähler moduli is achieved by tuning $\left|W_{0}\right| \ll M_{\mathrm{Pl}}^{3}$, where $M_{\mathrm{Pl}}$ is the reduced Planck mass. On the other hand, even if $\left|W_{0}\right| \sim \mathcal{O}\left(M_{\mathrm{Pl}}^{3}\right)$, the LVS works for CY manifolds with the negative Euler number, $\chi=2\left(h^{1,1}-h^{2,1}\right)<0$, i.e., $h^{2,1}>h^{1,1}>1 .^{2}$ Note that stringy modes and KaluzaKlein modes should be sufficiently heavier than all the moduli fields to justify description of the low-energy effective field theory.

In this paper, along the line of Ref. [10], we propose a new type of Kähler moduli stabilization mechanisms in type IIB superstring theory on CY orientifolds with the positive Euler number $\chi>0$, namely $h^{1,1}>h^{2,1}>1$. In Ref. [11], it was discussed that the overall volume modulus can be stabilized perturbatively by one-loop corrections to the Kähler potential. In that scenario, one needs a certain amount of fine-tuning of complex structure moduli to realize the Kähler moduli stabilization. In contrast to Ref. [11], here we focus on the radiative corrections due to the sparticles living on D7-branes wrapping a divisor of CY. Such corrections generate the potential of overall volume modulus through the Coleman-Weinberg potential [12]. Since the soft masses are functions of only the overall volume modulus, the overall Kähler modulus can be perturbatively stabilized at a sufficiently large volume region without tuning $W_{0}$. In this large volume region, the

\footnotetext{
${ }^{2}$ For the LVS with zero or positive Euler number, see Ref. [9].
} 
string axion associated with the overall volume modulus remains light and it could be a candidate of dark matter. Other Kähler moduli could be stabilized by the nonperturbative effects and/or moduli-dependent $D$-terms. (See, e.g., Refs. [13].) We find that stringy and KaluzaKlein modes are sufficiently heavier than sparticles and Kähler moduli at the anti-de Sitter minimum. We can uplift the anti-de Sitter vacuum to the de Sitter vacuum by introducing anti-D branes, and such uplifting does not change the behavior of the moduli stabilization.

The remaining paper is organized as follows. In Sec. II, we discuss the stabilization of overall Kähler modulus without uplifting terms in type IIB superstring theory on CY orientifolds with D7-branes. We add the uplifting term in the setup of Sec. III to achieve a tiny cosmological constant. We study the modulus potential with the uplifting terms analytically and numerically in Secs. III A and III B, respectively. Other Kähler moduli can be stabilized by two scenarios. One is due to moduli-dependent $D$-terms as discussed in Sec. III C, and the other is due to nonperturbative effects as discussed in Sec. III D. Also, in Sec. III E, we show that the nonperturbative effects generate the mass of ultralight axion associated with the volume modulus. Finally, Sec. IV is devoted to the conclusion.

\section{KÄHLER MODULI STABILIZATION WITHOUT UPLIFTING}

\section{A. Setup}

Let us consider the stabilization of closed string moduli on the basis of type IIB superstring theory on CY orientifolds with D7-branes. For a general class of CY threefolds, the total Kähler potential at the leading order of $\alpha^{\prime}$ is obtained through Kaluza-Klein reduction of type IIB supergravity action $[14,15]$,

$K=-2 M_{\mathrm{Pl}}^{2} \ln \left(\mathcal{V}+\frac{\xi}{2}\right)+M_{\mathrm{Pl}}^{2} K(S, U)+Z_{a \bar{a}}^{(i)}\left|Q_{a}^{(i)}\right|^{2}$,

where $\mathcal{V}$ is the volume of CY manifold in Einstein frame measured by the string length $l_{s}=2 \pi \sqrt{\alpha^{\prime}}, K(S, U)$ represents the Kähler potential of dilaton $S$ and complex structure moduli $U$. The leading $\alpha^{\prime}$-correction is characterized by $\xi=-\frac{\zeta(3) \chi}{2(2 \pi)^{3} g_{s}^{3 / 2}}$ where $\chi$ is the Euler number of CY and $g_{s}$ is the string coupling [14]. Here, $Q_{a}^{(i)}$ are the matter fields living on D7-branes wrapping the divisor $D_{i} \in H_{4}(\mathrm{CY}, \mathbb{Z})$ and their Kähler metrics $Z_{a \bar{a}}^{(i)}$ are given by

$$
Z_{a \bar{a}}^{(i)}=\left(T_{i}+\bar{T}_{i}\right)^{-n_{i}^{a}},
$$

with $n_{i}^{a}$ being the modular weights and $T_{i}=\tau_{i}+i \sigma_{i}$ with $i \in\left\{1,2, \ldots, h_{+}^{1,1}\right\}$ denotes the Kähler modulus. ${ }^{3}$ Also, the

\footnotetext{
${ }^{3}$ In this paper, we consider the orientifold projection to realize $h_{-}^{1,1}=0$, namely $h^{1,1}=h_{+}^{1,1}$.
}

gaugino fields couple to the Kähler moduli through the gauge kinetic function

$$
f=\frac{T_{i}}{2 \pi} .
$$

To stabilize the complex structure moduli $U$ and dilaton $S$, we consider the following superpotential induced by imaginary self-dual three-form fluxes [4],

$$
W=W_{\text {flux }}(S, U) .
$$

By the flux-induced superpotential, all the complex structure moduli and dilaton fields are stabilized at the compactification scale [3]. When $W_{0}=\left\langle W_{\text {flux }}\right\rangle \neq 0$ at the minima of the complex structure moduli and dilaton, the scalar potential is nonvanishing because of the breaking of the so-called no-scale structure [14]

$$
\begin{aligned}
V_{\alpha^{\prime}} & =e^{\langle K(S, U)\rangle}\left(K^{T_{i} \bar{T}_{j}} K_{T_{i}} K_{\bar{T}_{j}}-3\right) \frac{\left|W_{0}\right|^{2}}{M_{\mathrm{Pl}}^{2}} \\
& \simeq e^{\langle K(S, U)\rangle} \frac{3 \xi}{4 \mathcal{V}^{3}} \frac{\left|W_{0}\right|^{2}}{M_{\mathrm{Pl}}^{2}},
\end{aligned}
$$

where $K_{T_{i}}=\partial_{T_{i}} K$ and $K^{T_{i} \bar{T}_{j}}$ is the inverse of Kähler metric $K_{T_{i} \bar{T}_{j}}=\partial_{T_{i}} \partial_{\bar{T}_{j}} K$. The nonvanishing $F$-term of the Kähler modulus

$$
F^{T_{i}}=-e^{K /\left(2 M_{\mathrm{Pl}}^{2}\right)} K^{T_{i} \bar{T}_{j}} K_{\bar{T}_{j}} \frac{\bar{W}_{0}}{M_{\mathrm{Pl}}},
$$

generates the soft scalar masses and gaugino masses [16],

$$
\begin{aligned}
m_{a}^{2} & =\frac{V_{\alpha^{\prime}}}{M_{\mathrm{Pl}}^{2}}+m_{3 / 2}^{2}-\left(\partial_{T_{i}} \partial_{\bar{T}_{j}} \ln \left(Z_{a \bar{a}}^{(i)}\right)\right) \frac{F^{T_{i}} \bar{F}^{T_{j}}}{M_{\mathrm{Pl}}^{2}} \\
& =\frac{V_{\alpha^{\prime}}}{M_{\mathrm{Pl}}^{2}}+m_{3 / 2}^{2}-\frac{n_{i}^{a}}{M_{\mathrm{Pl}}^{2}}\left|\frac{F^{T_{i}}}{T_{i}+\bar{T}_{i}}\right|^{2} \\
& \simeq \frac{V_{\alpha^{\prime}}}{M_{\mathrm{Pl}}^{2}}+\left(1-n_{i}^{a}\right) m_{3 / 2}^{2} \simeq\left(1-n_{i}^{a}\right) m_{3 / 2}^{2}, \\
M_{f} & =\frac{F^{T_{i}}}{M_{\mathrm{Pl}}} \partial_{T_{i}} \ln \operatorname{Re}(f) \\
& =M_{\mathrm{Pl}}^{-1} \frac{F^{T_{i}}}{T_{i}+\bar{T}_{i}} \simeq \frac{e^{\langle K(S, U)\rangle / 2} \bar{W}_{0}}{\mathcal{V} M_{\mathrm{Pl}}^{2}} \simeq m_{3 / 2},
\end{aligned}
$$

with

$$
m_{3 / 2}=e^{K /\left(2 M_{\mathrm{Pl}}^{2}\right)} W_{0} M_{\mathrm{Pl}}^{-2} \simeq \frac{e^{\langle K(S, U)\rangle / 2} W_{0}}{\mathcal{V} M_{\mathrm{Pl}}^{2}},
$$

evaluated in the large volume region. ${ }^{4}$ Here, we evaluate $\frac{F_{i}^{T_{i}}}{T_{i}+\bar{T}_{i}} \simeq-e^{K /\left(2 M_{\mathrm{Pl}}^{2}\right)} \frac{\bar{W}_{0}}{M_{\mathrm{Pl}}} \simeq-m_{3 / 2} M_{\mathrm{Pl}}$, which is satisfied in a

\footnotetext{
${ }^{4}$ We take $W_{0}$ as a real constant for simplicity and discuss the case with $n_{i}^{a}=0$ later.
} 
general class of CY threefolds with a sufficiently large volume. When these sparticles contribute to the radiative corrections, the 1-loop Coleman-Weinberg $(\mathrm{CW})$ potential is given by

$$
\begin{aligned}
V_{\mathrm{CW}} & =\frac{1}{32 \pi^{2}} \int^{\Lambda^{2}} d k^{2} k^{2} \mathrm{~S} \operatorname{Tr} \ln \left(k^{2}+M^{2}\right) \\
& =\frac{1}{32 \pi^{2}}\left\{\Lambda^{2} \mathrm{~S} \operatorname{Tr}\left(M^{2}\right)+\frac{1}{2} \mathrm{~S} \operatorname{Tr} M^{4}\left[\ln \left(\frac{M^{2}}{\Lambda^{2}}\right)-\frac{1}{2}+\mathcal{O}\left(\frac{M^{2}}{\Lambda^{2}}\right)\right]\right\} \\
& \simeq \frac{c_{1}}{32 \pi^{2}} \Lambda^{2} m_{3 / 2}^{2}+\sum_{a} \frac{c_{b}^{a}}{64 \pi^{2}} m_{a}^{4}\left[\ln \left(\frac{m_{a}^{2}}{\Lambda^{2}}\right)-\frac{1}{2}\right]+\frac{-2 c_{f}-4}{64 \pi^{2}} m_{3 / 2}^{4}\left[\ln \left(\frac{m_{3 / 2}^{2}}{\Lambda^{2}}\right)-\frac{1}{2}\right]+\frac{32}{64 \pi^{2}} m_{3 / 2}^{4}\left[\ln \left(\frac{4 m_{3 / 2}^{2}}{\Lambda^{2}}\right)-\frac{1}{2}\right],
\end{aligned}
$$

where $M$ characterizes the mass matrix for canonically normalized bosons and fermions, and

$$
c_{1}=\sum_{a} c_{b}^{a}\left(1-n_{i}^{a}\right)-2 c_{f}+4
$$

with $c_{b}^{a}$ and $c_{f}$ being the multiplicities of bosons $Q_{a}^{(i) 5}$ and fermions, respectively. Here, we take the limit $M^{2} / \Lambda^{2} \ll 1$, where our description of low-energy effective theory is valid and the supertrace is defined as

$\mathrm{STr} f\left(M^{2}\right)=\operatorname{tr} f\left(m_{a}^{2}\right)-2 \operatorname{tr} f\left(M_{f}^{2}\right)-4 f\left(m_{3 / 2}^{2}\right)+2 f\left(4 m_{3 / 2}^{2}\right)$,

for an arbitrary function $f$. We consider the contribution of ghosts in the gauge $\sum_{\mu=0}^{3} \gamma^{\mu} \psi_{\mu}=0$, where $\psi_{\mu}$ and $\gamma^{\mu}$ are the gravitino and four-dimensional gamma-matrices, respectively. (For more details, see Refs. [17].) Obviously, there is no contribution from sfermion fields with $n_{i}^{a}=1$.

For example, in the minimal supersymmetric standard model (MSSM) with three generations of right-handed (s) neutrinos, those multiplicities become

$$
c_{b}^{(\mathrm{MSSM})}=\sum_{a} c_{b}^{a}=49+3=52, \quad c_{f}^{(\mathrm{MSSM})}=12 .
$$

On the other hand, when the visible sector consists of the MSSM with singlets and multi-Higgs doublets, the multiplicities are given by

$$
c_{b}=c_{b}^{(\mathrm{MSSM})}+4\left(n_{H}-1\right)+n_{S}, \quad c_{f}^{(\mathrm{MSSM})}=12,
$$

where $n_{H}$ and $n_{S}$ denote the numbers of multi-Higgs doublets and singlet fields. Note that the following moduli stabilization is also applicable to the scenario where sparticles living on hidden D7-branes contribute to the radiative corrections. If there exist multiple $\mathrm{D} 7_{i}$-branes

\footnotetext{
${ }^{5}$ We use the same notation of chiral superfields $Q_{a}$ and their lowest components.
}

wrapping the divisor $D_{i}$, the matter fields $Q_{a}^{(i)}$ and gauginos living on $\mathrm{D} 7{ }_{i}$-branes contribute to the radiative corrections through the soft masses (7). For sake of simplicity, we discuss the D7-branes wrapping only the divisor $D_{i}$ with volume $\tau_{i}$ in the following analysis.

To justify this low-energy effective action, the soft masses and masses of Kähler moduli $m_{T}$ should be smaller than those of Kaluza-Klein (KK) modes, stringy modes, complex structure and dilaton moduli, namely

$$
m_{T}, m_{3 / 2}<m_{U}, m_{S}<m_{\mathrm{KK}}<m_{\mathrm{st}}<M_{\mathrm{Pl}},
$$

and their typical masses are given by

$m_{S, U} \simeq N m_{3 / 2}, \quad m_{\mathrm{KK}} \simeq \frac{\sqrt{\pi}}{\mathcal{V}^{2 / 3}} M_{\mathrm{Pl}}, \quad m_{\mathrm{st}} \simeq \frac{\sqrt{\pi} g_{s}^{1 / 4}}{\mathcal{V}^{1 / 2}} M_{\mathrm{Pl}}$,

with $N>1$ being a parameter determined by three-form fluxes. Note that we have taken the string-frame volume $\mathcal{V}_{s}=\mathcal{V} g_{s}^{3 / 2} \simeq(2 \pi R)^{6} l_{s}^{6}$, where $R$ is the typical length of $C Y$ in string units. Since the cutoff scale is a physical quantity in the nonrenormalizable theory, there exist several options for the cutoff scale. For concreteness, we take the cutoff scale as the typical masses of $\mathrm{KK}$ modes in the following analysis, since there exists $N=2$ supersymmetry above the KK scale. The CW potential is then valid below the cutoff scale. The string loop corrections to the Kähler potential was discussed in Ref. [11], and they can also be interpreted as the 1-loop CW potentials [18]. However, in this paper, we focus on the situation where sparticle contributions dominate over the 1-loop CW potential. ${ }^{6}$ Since the following analysis requires that a relatively large number of sparticle contribute to stabilize the $\mathrm{CY}$ volume, they could dominate over the string loop corrections to the Kähler potential controlled by the string coupling.

\footnotetext{
${ }^{6}$ The moduli stabilization involving the string loop corrections to the Kähler potential is discussed for CY threefolds with negative Euler number [19] and zero or positive Euler number [9].
} 


\section{B. Stabilization of overall volume modulus}

In the following analysis, we first consider the large volume region $\mathcal{V} \rightarrow \infty$, where $\tau_{i} \rightarrow \infty$ with $i=$ $1,2, \ldots, h^{1,1}$ for all of the divisors. (In the LVS, some of the divisor volumes are not extremely large in string units [6].) When there exist the D-brane instanton effects or gaugino condensation on D7-branes, the nonperturbative superpotential for the Kähler moduli is generated as

$$
W_{\text {non }}=\sum_{i} A_{i} e^{-a_{i} T_{i}}
$$

where $A_{i}$ are functions of the complex structure moduli and $a_{i}=2 \pi$ for the brane instanton and $a_{i}=2 \pi / N$ for the gaugino condensation on $N$ stacks of D7-branes wrapping the divisor with volume $\tau_{i}$. In the moduli space $\tau_{i} \rightarrow \infty$, these nonperturbative effects are negligible and the total scalar potential consists of the leading $\alpha^{\prime}$-corrections and radiative corrections. We come back to these nonperturbative effects in Secs. III C-III E.

Including the radiative corrections due to sfermions and gauginos, we can write the scalar potential by

$$
\begin{aligned}
V_{F}= & V_{\alpha^{\prime}}+V_{\mathrm{CW}} \\
= & e^{\langle K(S, U)\rangle} \frac{3 \xi}{4 \mathcal{V}^{3}} \frac{\left|W_{0}\right|^{2}}{M_{\mathrm{Pl}}^{2}}+\frac{c_{1}}{32 \pi^{2}} \Lambda^{2} m_{3 / 2}^{2} \\
& +\sum_{a} \frac{c_{b}^{a}}{64 \pi^{2}} m_{a}^{4}\left[\ln \left(\frac{m_{a}^{2}}{\Lambda^{2}}\right)-\frac{1}{2}\right] \\
& +\frac{-2 c_{f}-4}{64 \pi^{2}} m_{3 / 2}^{4}\left[\ln \left(\frac{m_{3 / 2}^{2}}{\Lambda^{2}}\right)-\frac{1}{2}\right] \\
& +\frac{32}{64 \pi^{2}} m_{3 / 2}^{4}\left[\ln \left(\frac{4 m_{3 / 2}^{2}}{\Lambda^{2}}\right)-\frac{1}{2}\right] .
\end{aligned}
$$

It turns out that the $F$-term scalar potential is a function of only the overall volume $\mathcal{V}$. In the large volume regime $\mathcal{V} \gg 1$, the above scalar potential is further approximated as

$V_{F} \simeq e^{\langle K(S, U)\rangle} \frac{3 \xi}{4 \mathcal{V}^{3}} \frac{\left|W_{0}\right|^{2}}{M_{\mathrm{Pl}}^{2}}+\frac{c_{1}}{32 \pi^{2}} \Lambda^{2} \frac{e^{\langle K(S, U)\rangle}\left|W_{0}\right|^{2}}{\mathcal{V}^{2} M_{\mathrm{Pl}}^{4}}$

Let us fix the cutoff scale as the KK scale and consider the following units

$$
\Lambda=m_{\mathrm{KK}}=\frac{\sqrt{\pi}}{\mathcal{V}^{2 / 3}} M_{\mathrm{Pl}}=1,
$$

and then the dimensional quantities are rewritten as

$$
\begin{aligned}
M_{\mathrm{Pl}} & =\frac{\mathcal{V}^{2 / 3}}{\sqrt{\pi}}, \\
W_{0} & \equiv \hat{W}_{0} M_{\mathrm{Pl}}^{3}=\hat{W}_{0} \frac{\mathcal{V}^{2}}{\pi^{3 / 2}} .
\end{aligned}
$$

In units of $m_{\mathrm{KK}}$, the approximated scalar potential reduces to

$$
V_{F} \simeq e^{\langle K(S, U)\rangle} \frac{\left|\hat{W}_{0}\right|^{2}}{\pi^{2}}\left[\frac{3 \xi}{4 \mathcal{V}^{1 / 3}}+\frac{c_{1}}{32 \pi^{2}} \frac{\pi}{\mathcal{V}^{2 / 3}}\right]
$$

From this scalar potential, we aim to find the minimum of $\mathcal{V}$ in the large volume regime $\mathcal{V} \gg 1$. The extremal condition of modulus field $\left\langle\partial_{\mathcal{V}} V_{F}\right\rangle=0$ in the large volume limit,

$\partial_{\mathcal{V}} V_{F} \simeq e^{\langle K(S, U)\rangle} \frac{\left|\hat{W}_{0}\right|^{2}}{\pi^{2}}\left[-\frac{\xi}{4 \mathcal{V}^{4 / 3}}-\frac{c_{1}}{48 \pi^{2}} \frac{\pi}{\mathcal{V}^{5 / 3}}\right]=0$,

is satisfied at

$$
\langle\mathcal{V}\rangle \simeq 18664\left(-\frac{c_{1} / \xi}{10^{3}}\right)^{3}
$$

in string units. In particular, when the $\mathrm{CY}$ volume is dominated by a single Kähler modulus $\mathcal{V} \simeq \kappa(T+\bar{T})^{3 / 2}$ with $\kappa$ being a real positive constant, the value of overall volume modulus is evaluated as

$$
\langle\tau\rangle \simeq 351.8\left(-\frac{c_{1} / \xi}{10^{3}}\right)^{2} \kappa^{-2 / 3}
$$

where $T \equiv \tau+i \sigma$. We find that the ratio $c_{1} / \xi$ should be negative and larger than $10^{2-3}$ such that the Kähler moduli space resides in the physical domain.

Furthermore, the positivity of $\left\langle\partial_{\mathcal{V}} \partial_{\mathcal{V}} V_{F}\right\rangle$,

$$
\partial_{\mathcal{V}} \partial_{\mathcal{V}} V_{F} \simeq 9.14 \times 10^{8} e^{\langle K(S, U)\rangle}\left|\hat{W}_{0}\right|^{2} \frac{\xi^{8}}{c_{1}^{7}}>0
$$

requires that $c_{1}$ should be positive. That indicates the negative $\xi$ from Eq. (23). Obviously, the sfermions with $n_{i}^{a}=1$ have no contribution in the potential. Thus, it turns out that the case with $n_{i}^{a}=1$ for all sfermions is prohibited, because such a case corresponds to the negative $c_{1}$ from Eq. (10). In addition, the potential energy becomes negative at this minimum, namely anti-de Sitter minimum,

$$
\left\langle V_{F}\right\rangle \simeq 1.4 \times 10^{-3} e^{\langle K(S, U)\rangle}\left|\hat{W}_{0}\right|^{2}\left(-\frac{10^{3}}{c_{1} / \xi}\right) \xi<0,
$$

where $\xi$ is negative. 
At this minimum, the mass scales of typical modes become

$$
\begin{aligned}
m_{a} & \simeq M_{f} \simeq m_{3 / 2}=\frac{e^{\langle K(S, U)\rangle / 2}\left|\hat{W}_{0}\right|}{\mathcal{V}} M_{\mathrm{Pl}} \\
& \simeq 2.1 \times 10^{-2}\left(-\frac{10^{3}}{c_{1} / \xi}\right) e^{\langle K(S, U)\rangle / 2}\left|\hat{W}_{0}\right| m_{\mathrm{KK}} \\
m_{U, S} & =N m_{3 / 2}=2.1 \times 10^{-2} N\left(-\frac{10^{3}}{c_{1} / \xi}\right) e^{\langle K(S, U)\rangle / 2}\left|\hat{W}_{0}\right| m_{\mathrm{KK}}, \\
m_{\mathrm{KK}} & =\frac{\sqrt{\pi}}{\mathcal{V}^{2 / 3}} M_{\mathrm{Pl}}=1 \\
m_{\mathrm{st}} & =\frac{\sqrt{\pi} g_{s}^{1 / 4}}{\mathcal{V}^{1 / 2}} M_{\mathrm{Pl}}=2.9\left(\frac{g_{s}}{10^{-1}}\right)^{1 / 4}\left(-\frac{10^{3}}{c_{1} / \xi}\right)^{1 / 2} m_{\mathrm{KK}} \\
M_{\mathrm{Pl}} & =397\left(-\frac{c_{1} / \xi}{10^{3}}\right)^{2} m_{\mathrm{KK}} \cdot
\end{aligned}
$$

Especially, when the CY volume is dominated by a single Kähler modulus, the mass scale of canonically-normalized overall Kähler modulus is

$m_{\tau} \simeq 1.1 \times 10^{-3} e^{\langle K(S, U)\rangle / 2}\left|\hat{W}_{0}\right|\left(-\frac{10^{3}}{c_{1} / \xi}\right)^{3 / 2}(-\xi)^{1 / 2} m_{\mathrm{KK}}$.

In this way, we find that the volume modulus can be perturbatively stabilized at the anti-de Sitter minimum, where four-dimensional supergravity description is reliable. The nonperturbative effects for the overall Kähler modulus are suppressed enough by the value of Kähler modulus, $e^{-2 \pi \tau} \ll 1$, but it generates the potential of remaining massless axion as discussed in Sec. IIIE. However, the negative $\xi$ indicates that we have to consider the CY threefolds with the positive Euler number, namely a large number of Kähler moduli compared with that of complex structure moduli. ${ }^{7}$ (In the LVS, the negative Euler number is required to realize the large CY volume.) Since such CY threefolds with the positive Euler number account for half of the whole CY threefolds in the sense of mirror symmetry, the CYs with the positive Euler number are not so restricted manifolds, but generic. The O7-plane contribution will also help us to change the number of CY Euler number in a weak coupling limit [21]. In Secs. III C and III D, we discuss the stabilization of other Kähler moduli. Although for a generic value of $e^{\langle K(S, U)\rangle / 2}\left|\hat{W}_{0}\right|$, the overall volume modulus can be stabilized at the anti-de Sitter minimum in a way similar to the LVS, we require the relatively large $c_{1}$ to realize the large volume of CY, e.g., $c_{1} \simeq \mathcal{O}\left(10^{2}\right)$ for $|\xi| \simeq \mathcal{O}\left(10^{-1}\right)$. Such a situation is easily realized by the models with multi-Higgs doublets and

\footnotetext{
${ }^{7}$ Our result is similar to Ref. [20], where the higher-derivative corrections are taken into account.
}

singlets via Eq. (13), and such models often appear from concrete string model constructions. (For the string model building, see e.g., Refs. [22,23].)

Finally, we comment on the case with no leading $\alpha^{\prime}$ corrections, which corresponds to the CY manifold with the vanishing Euler number, i.e., $\chi=0$. Then, the scalar potential consists of only the $\mathrm{CW}$ potential,

$V_{\mathrm{CW}}=\frac{c_{1}}{32 \pi^{2}} \Lambda^{2} m_{3 / 2}^{2}+\frac{c_{2}}{64 \pi^{2}} m_{3 / 2}^{4}\left[\ln \left(\frac{m_{3 / 2}^{2}}{\Lambda^{2}}\right)\right]+\frac{c_{3}}{64 \pi^{2}} m_{3 / 2}^{4}$,

with

$$
\begin{aligned}
c_{2} \equiv & \sum_{a} c_{b}^{a}\left(1-n_{i}^{a}\right)^{2}-2 c_{f}-4+32, \\
c_{3} \equiv & \sum_{a}\left[c_{b}^{a}\left(1-n_{i}^{a}\right)^{2}\left(-\frac{1}{2}+\ln \left(1-n_{i}^{a}\right)\right)\right]+c_{f} \\
& +2+32\left(\ln 4-\frac{1}{2}\right) .
\end{aligned}
$$

It can be rewritten in units of $\Lambda=m_{\mathrm{KK}}=1$,

$$
\begin{aligned}
V_{\mathrm{CW}} & \simeq e^{\langle K(S, U)\rangle} \frac{\left|\hat{W}_{0}\right|^{2}}{\left(32 \pi^{2}\right) \pi^{2}}\left[c_{1} \frac{\pi}{\mathcal{V}^{2 / 3}}+c_{2} \frac{e^{\langle K(S, U)\rangle}\left|\hat{W}_{0}\right|^{2}}{\mathcal{V}^{4 / 3}}\right. \\
& \left.\times \ln \left(\frac{e^{\langle K(S, U)\rangle / 2} \hat{W}_{0}}{\mathcal{V}^{1 / 3} \sqrt{\pi}}\right)+c_{3} \frac{e^{\langle K(S, U)\rangle}\left|\hat{W}_{0}\right|^{2}}{2 \mathcal{V}^{4 / 3}}\right] \\
\simeq & e^{\langle K(S, U)\rangle} \frac{\left|\hat{W}_{0}\right|^{2}}{\left(32 \pi^{2}\right) \pi^{2}}\left[c_{1} \frac{\pi}{\mathcal{V}^{2 / 3}}-\frac{c_{2}}{3} \frac{e^{\langle K(S, U)\rangle}\left|\hat{W}_{0}\right|^{2}}{\mathcal{V}^{4 / 3}} \ln (\mathcal{V})\right],
\end{aligned}
$$

in the large volume regime $\mathcal{V} \gg 1$ and $e^{\langle K(S, U)\rangle / 2} \hat{W}_{0} \sim \mathcal{O}(1)$. When $c_{2}$ is almost the same order of $c_{1}$, we cannot find the minimum with a sufficiently large $\mathrm{CY}$ volume.

\section{Numerical estimation}

In this section, we numerically analyze the stabilization of moduli fields. The unapproximated scalar potential of the overall volume modulus (17) in units of $\Lambda=m_{\mathrm{KK}}$ is drawn as functions of $\mathcal{V}$ and $\tau$ in Fig. 1, where we set the following parameters,

$$
\begin{aligned}
\xi & =-0.1, \quad e^{\langle K(S, U)\rangle / 2}\left|\hat{W}_{0}\right|=1, \quad n_{i}^{a}=0, \\
\kappa & =1, \quad c_{b}=120, \quad c_{f}=12 .
\end{aligned}
$$

At this vacuum in Fig. 1, the CY volume is determined by

$$
\langle\mathcal{V}\rangle \simeq 18433
$$



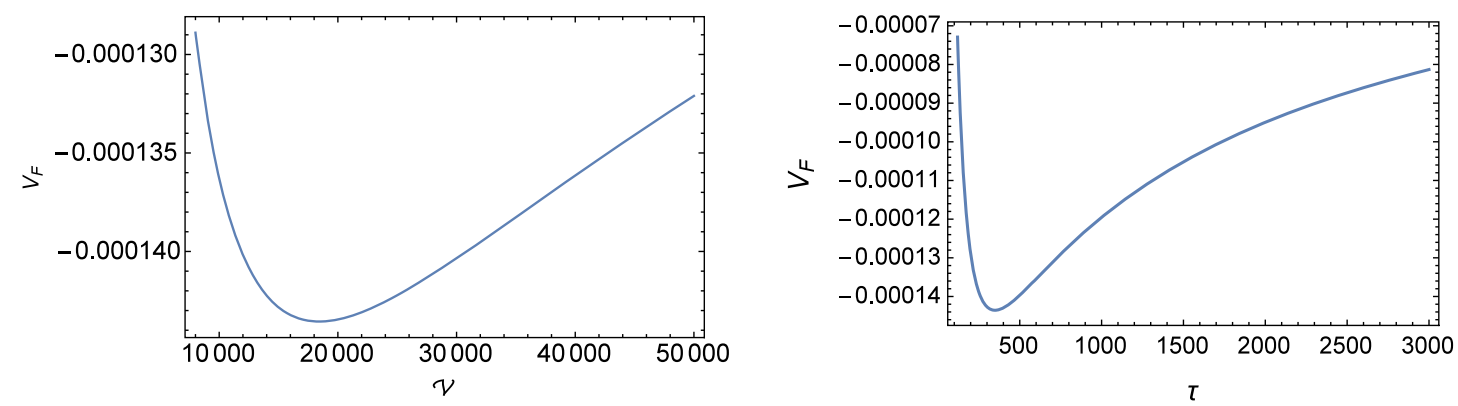

FIG. 1. The scalar potential (17) as functions of CY volume $\mathcal{V}$ and noncanonically normalized modulus $\tau$, where the CY volume is approximated as $\mathcal{V}=(T+\bar{T})^{3 / 2}$ with $T=\tau+i \sigma$ in the right panel.

as shown in the left panel and the vacuum expectation value of overall Kähler modulus is

$$
\langle\tau\rangle \simeq 348.9
$$

as shown in the right panel. The mass scales of typical modes become

$$
\begin{aligned}
m_{i} & =M_{f}=m_{3 / 2}=2.1 \times 10^{-2} m_{\mathrm{KK}}, \\
m_{U, S} & =N m_{3 / 2}=2.1 \times 10^{-2} N m_{\mathrm{KK}}, \\
m_{\mathrm{KK}} & =1, \\
m_{\mathrm{st}} & =2.9\left(\frac{g_{s}}{10^{-1}}\right)^{1 / 4} m_{\mathrm{KK}}, \\
M_{\mathrm{Pl}} & =394 m_{\mathrm{KK}} .
\end{aligned}
$$

In addition, the mass scale of canonically-normalized overall Kähler modulus is

$$
m_{\tau} \simeq 3.5 \times 10^{-4} m_{\mathrm{KK}},
$$

when the volume of CY is dominated by the single Kähler modulus $T=\tau+i \sigma$. Hence, even when we involve the next leading terms in the scalar potential, which are volume-suppressed, the numerical result is in agreement with the analytical one in Sec. II B.

\section{KÄHLER MODULI STABILIZATION WITH UPLIFTING}

In this section, we introduce the uplifting term to achieve a tiny cosmological constant. In particular, we focus on the anti-D3 branes to uplift the anti-de Sitter minimum to the de Sitter minimum.

\section{A. Analytical estimation}

When there exist anti-D3 branes at certain moduli space of CY manifold, the uplifting term is given by

$$
V_{\text {up }}=\frac{\epsilon}{\mathcal{V}^{2}} M_{\mathrm{Pl}}^{4},
$$

where $\epsilon$ is a real constant suppressed by the warp factor. Hence, the total scalar potential is a sum of $V_{F}$ in Eq. (17) and $V_{\text {up }}$.

Similar to the analysis in Sec. II, we aim to find the minimum of modulus field analytically. In the large volume regime, the above scalar potential in units of $m_{\mathrm{KK}}$ is simplified as

$$
\begin{aligned}
V & =V_{F}+V_{\text {up }} \\
& \simeq e^{\langle K(S, U)\rangle} \frac{\left|\hat{W}_{0}\right|^{2}}{\pi^{2}}\left[\frac{3 \xi}{4 \mathcal{V}^{1 / 3}}+\frac{c_{1}}{32 \pi^{2}} \frac{\pi}{\mathcal{V}^{2 / 3}}\right]+\frac{\epsilon}{\pi^{2}} \mathcal{V}^{2 / 3},
\end{aligned}
$$

from which the minimum of overall volume modulus is determined by solving $\partial_{\mathcal{V}} V=0$,

$$
\langle\mathcal{V}\rangle \simeq 5530\left(-\frac{c_{1} / \xi}{10^{3}}\right)^{3},
$$

and $\epsilon$ is chosen such that $V \simeq 0$,

$$
\epsilon \simeq 3.4 \times 10^{-5} e^{\langle K(S, U)\rangle / 2}\left|\hat{W}_{0}\right|^{2}(-\xi)\left(-\frac{10^{3}}{c_{1} / \xi}\right)^{3} .
$$

In particular, when the CY volume is dominated by a single Kähler modulus $\mathcal{V} \simeq \kappa(T+\bar{T})^{3 / 2}$ with $\kappa$ being a real positive constant, the modulus value at this minimum is evaluated as

$$
\langle\tau\rangle \simeq 156\left(-\frac{c_{1} / \xi}{10^{3}}\right)^{2} \kappa^{-2 / 3} .
$$

It turns out that the deviation from the SUSY-breaking antide Sitter minimum to the uplifted minimum is estimated as

$$
\frac{\left.\langle\tau\rangle\right|_{\text {anti-de Sitter }}-\left.\langle\tau\rangle\right|_{\text {de Sitter }}}{\left.\langle\tau\rangle\right|_{\text {anti-de Sitter }}} \simeq 0.56
$$

Our scalar potential still resides in the supergravitycontrolled regime, since the mass scales of typical modes have a desirable hierarchical structure, 
TABLE I. The volume of CY $(\mathcal{V})$ and divisor $(\tau)$, and typical scales of several modes, setting $e^{\langle K(S, U)\rangle / 2}\left|\hat{W}_{0}\right|=1, g_{s}=0.1$, and $\xi=-0.1$.

\begin{tabular}{lccc}
\hline \hline Scale & $c_{1}=50$ & $c_{1}=100$ & $c_{1}=1000$ \\
\hline $\mathcal{V}$ & 691 & 5530 & $5.5 \times 10^{6}$ \\
$\tau$ & $39 \kappa^{-2 / 3}$ & $156 \kappa^{-2 / 3}$ & $1.6 \times 10^{4} \kappa^{-2 / 3}$ \\
$m_{\tau}[\mathrm{GeV}]$ & $1.7 \times 10^{14}$ & $1.5 \times 10^{13}$ & $4.8 \times 10^{9}$ \\
$m_{3 / 2}[\mathrm{GeV}]$ & $3.5 \times 10^{15}$ & $4.4 \times 10^{14}$ & $4.4 \times 10^{11}$ \\
$m_{U, S}[\mathrm{GeV}]$ & $3.5 N \times 10^{15}$ & $4.4 N \times 10^{14}$ & $4.4 N \times 10^{11}$ \\
$m_{\mathrm{KK}}[\mathrm{GeV}]$ & $5.5 \times 10^{16}$ & $1.4 \times 10^{16}$ & $1.4 \times 10^{14}$ \\
$m_{\mathrm{st}}[\mathrm{GeV}]$ & $9.1 \times 10^{16}$ & $3.2 \times 10^{16}$ & $1.0 \times 10^{15}$ \\
$M_{\mathrm{Pl}}[\mathrm{GeV}]$ & $2.4 \times 10^{18}$ & $2.4 \times 10^{18}$ & $2.4 \times 10^{18}$ \\
\hline \hline
\end{tabular}

$$
\begin{aligned}
m_{a} & \simeq M_{f} \simeq m_{3 / 2}=\frac{e^{\langle K(S, U)\rangle / 2}\left|\hat{W}_{0}\right|}{\langle\mathcal{V}\rangle} M_{\mathrm{Pl}} \\
& \simeq 3.2 \times 10^{-2}\left(-\frac{10^{3}}{c_{1} / \xi}\right) e^{\langle K(S, U)\rangle / 2}\left|\hat{W}_{0}\right| m_{\mathrm{KK}}, \\
m_{U, S} & =N m_{3 / 2}=3.2 \times 10^{-2} N\left(-\frac{10^{3}}{c_{1} / \xi}\right) e^{\langle K(S, U)\rangle / 2}\left|\hat{W}_{0}\right| m_{\mathrm{KK}}, \\
m_{\mathrm{KK}} & =\frac{\sqrt{\pi}}{\langle\mathcal{V}\rangle^{2 / 3}} M_{\mathrm{Pl}}=1 \\
m_{\mathrm{st}} & =\frac{\sqrt{\pi} g_{s}^{1 / 4}}{\langle\mathcal{V}\rangle^{1 / 2}} M_{\mathrm{Pl}}=2.4\left(\frac{g_{s}}{10^{-1}}\right)^{1 / 4}\left(-\frac{10^{3}}{c_{1} / \xi}\right)^{1 / 2} m_{\mathrm{KK}}, \\
M_{\mathrm{Pl}} & =176\left(-\frac{c_{1} / \xi}{10^{3}}\right)^{2} m_{\mathrm{KK}} .
\end{aligned}
$$

Furthermore, when the CY volume is dominated by a single Kähler modulus, the mass scale of canonically-normalized overall Kähler modulus is

$m_{\tau} \simeq 3.5 \times 10^{-3} e^{\langle K(S, U)\rangle / 2}\left|\hat{W}_{0}\right|\left(-\frac{10^{3}}{c_{1} / \xi}\right)^{3 / 2}(-\xi)^{1 / 2} m_{\mathrm{KK}}$

We find that the volume modulus is still stabilized at the supergravity-reliable de Sitter minimum, even after the anti-de Sitter minimum is uplifted to the de Sitter vacuum.

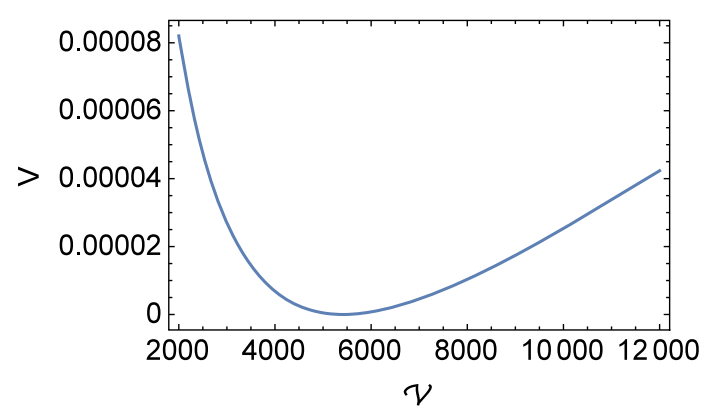

By setting $e^{\langle K(S, U)\rangle / 2}\left|\hat{W}_{0}\right|=1, g_{s}=0.1$, and $\xi=-0.1$, we list the volume of CY, the typical scales of gravitino, moduli fields, KK and stringy modes for several values of $c_{1}$ in Table I.

\section{B. Numerical estimation}

Next, let us numerically estimate the unapproximated scalar potential $V=V_{F}+V_{\text {up }}$ setting the same parameters as ones in Sec. II C and

$$
\epsilon \simeq 3.437 \times 10^{-6},
$$

under which the scalar potential with the uplifting term is drawn in Fig. 2.

In the left panel in Fig. 2, the CY volume is determined as

$$
\langle\mathcal{V}\rangle \simeq 5424
$$

and in the right panel, the vacuum expectation value of overall Kähler modulus is

$$
\langle\tau\rangle \simeq 154.4 .
$$

The mass scales of typical modes become

$$
\begin{aligned}
m_{i} & =M=m_{3 / 2}=3.2 \times 10^{-2} m_{\mathrm{KK}}, \\
m_{U, S} & =N m_{3 / 2}=3.2 \times 10^{-2} N m_{\mathrm{KK}}, \\
m_{\mathrm{KK}} & =1, \\
m_{\mathrm{st}} & =2.4\left(\frac{g_{s}}{10^{-1}}\right)^{1 / 4} m_{\mathrm{KK}}, \\
M_{\mathrm{Pl}} & =174 m_{\mathrm{KK}},
\end{aligned}
$$

and in addition the mass scale of canonically-normalized overall Kähler modulus is

$$
m_{\tau} \simeq 1.1 \times 10^{-3} m_{\mathrm{KK}} .
$$

Hence, even when we involve the next leading terms in the scalar potential, which are volume-suppressed, the numerical result is in agreement with the analytical one.

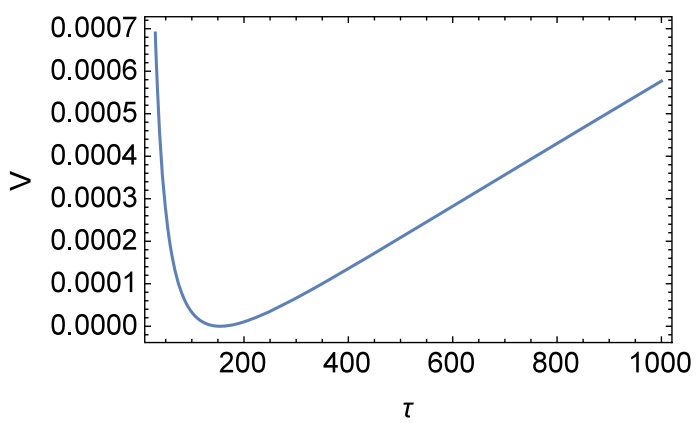

FIG. 2. The scalar potential in units of $\Lambda=m_{\mathrm{KK}}$ as functions of CY volume $\mathcal{V}$ and noncanonically normalized modulus $\tau$, where the CY volume is approximated as $\mathcal{V}=(T+\bar{T})^{3 / 2}$ with $T=\tau+i \sigma$ in the right panel. 


\section{Inclusion of the $D$-terms}

So far, we have not considered the nonperturbative effects for the Kähler moduli, since those are negligible in the large volume regime $\mathcal{V} \rightarrow \infty$, where $\tau_{i} \rightarrow \infty$ for all the volumes of divisors. To stabilize the Kähler moduli fields expect for the overall volume modulus, we consider the $D$-term potential induced by the Fayet-Iliopoulos (FI) term [13]. When the anomalous $U(1)$ symmetries appear on $\mathrm{D} 7_{i}$-branes wrapping the divisors $\tau_{i}$, the $D$-term potential is written by

$$
V_{D}^{\mathrm{D} 7}=\sum_{i} \frac{1}{\operatorname{Re}\left(f_{\mathrm{D} 7_{i}}\right)}\left(q_{T_{i}}^{\left(\mathrm{D} 7_{i}\right)} K_{T_{i}}-\sum_{m} q_{\varphi^{m}}^{\left(\mathrm{D} 7_{i}\right)}\left|\phi_{m}\right|^{2}\right)^{2},
$$

where $f_{\mathrm{D} 7_{i}}$ are the gauge kinetic functions of $\mathrm{D} 7_{i}$-branes, $q_{T_{i}}^{\left(\mathrm{D} 7_{i}\right)}$ represent the gauge fluxes and $\phi_{m}$ with U(1) charges $q_{\varphi^{m}}^{\left(\mathrm{D} 7_{i}\right)}$ are the canonically normalized matter fields living on D7 ${ }_{i}$-branes. In addition to these D7-brane contributions, anomalous $U(1)$ symmetries on the fractional D3-branes located at the singularities of CY also induce the $D$-terms

$$
V_{D}^{\mathrm{D} 3}=\sum_{k} \frac{1}{\operatorname{Re}\left(f_{\mathrm{D} 3_{k}}\right)}\left(q_{T_{k}}^{\left(\mathrm{D} 3_{k}\right)} K_{T_{k}}-\sum_{m} q_{\varphi^{m}}^{\left(\mathrm{D} 3_{k}\right)}\left|\varphi_{m}\right|^{2}\right)^{2},
$$

where $f_{\mathrm{D} 3_{k}}$ are the gauge kinetic functions of $\mathrm{D} 3_{k}$-branes with $k$ being the number of singularities, $q_{T_{k}}^{\left(\mathrm{D} 3_{k}\right)}$ represent the U(1) charges of moduli fields and $\varphi_{m}$ with U(1) charges $q_{\varphi^{m}}^{(\mathrm{D} 3)}$ are the canonically normalized matter fields living on the fractional $\mathrm{D}_{k}$-branes. In both cases, the anomalous $\mathrm{U}(1)$ gauge bosons eat the linear combination of string axions through the Stückelberg couplings and become massive at the compactification scale. In order not to spoil the stabilization of $F$-term potential, we require the vanishing $D$-term potential, $V_{D}^{\mathrm{D} 7}=V_{D}^{\mathrm{D} 3}=0$, namely

$q_{T_{i}}^{\left(\mathrm{D} 7_{i}\right)} K_{T_{i}}=\sum_{m} q_{\varphi^{m}}^{\left(\mathrm{D} 7_{i}\right)}\left|\phi_{m}\right|^{2}, \quad q_{T_{k}}^{\left(\mathrm{D} 3_{k}\right)} K_{T_{k}}=\sum_{m} q_{\varphi^{m}}^{\left(\mathrm{D} 3_{k}\right)}\left|\varphi_{m}\right|^{2}$, which fix the linear combination of closed string moduli and matter fields (open string moduli). In addition, we consider the $F$-term potential of matter fields,

$$
V_{F}^{\text {matter }}=m_{\Phi}^{2}|\Phi|^{2}+A_{\Phi}|\Phi|^{3}+\lambda_{\Phi}|\Phi|^{4},
$$

where $\Phi$ denotes $\phi_{m}$ and $\varphi_{m}, m_{\Phi}^{2}, A_{\Phi}, \lambda_{\Phi}$ are $T_{i}$-dependent functions. When $\Phi$ develops a nonvanishing value, the $D$-term and $F$-term contributions are possible to stabilize all the moduli fields except for the overall volume modulus, although it depends on a topology of CY manifold. We leave an explicit moduli stabilization for a future work, since it is difficult to analyze the topology of CY threefold with huge $h^{1,1}$. (For the model building in the LVS with small $h^{1,1}$, see Ref. [24].)

\section{Inclusion of the nonperturbative effects}

In contrast to the previous section, we study alternative stabilization scenario for Kähler moduli other than the overall volume modulus. We consider the large volume regime $\mathcal{V} \rightarrow \infty$, but the volume of one divisor $\tau_{s}$ is not extremely larger than the other Kähler moduli $\tau_{i}$, namely $\tau_{i} \gg \tau_{s}>1$ in string units. Note that we focus on the Kähler cone where $\tau_{s}$ is larger than the string length to suppress the $\mathrm{KK}$ and stringy corrections.

Let us assume the nonpertrubative superpotential with respect to $\tau_{s}$,

$$
W=W_{0}+A_{s} e^{-a_{s} T_{s}},
$$

where $W_{0}=\left\langle W_{\text {flux }}\right\rangle, A_{s}$ and $a_{s}$ are the constants depending on the origin of nonperturbative effects, e.g., $a_{s}=2 \pi$ for the brane instanton and $a_{s}=2 \pi / N$ for the gaugino condensation on $N$ stacks of D7-branes wrapping the divisor with volume $\tau_{s}$. To simplify the following analysis, we take both the $W_{0}$ and $A_{s}$ as real constants. From the Kähler potential (1) and superpotential (54), the $F$-term scalar potential is simply given by

$$
\begin{aligned}
V_{F} M_{\mathrm{Pl}}^{2}= & e^{K}\left(K_{i}^{T_{i} \bar{T}_{j}} D_{T_{i}} W D_{\bar{T}_{j}} \bar{W}-3\right)|W|^{2} \\
\simeq & \frac{e^{K(S, U)}}{\mathcal{V}^{2}}\left[K^{T_{s} \bar{T}_{s}} a_{s}^{2}\left|A_{s}\right|^{2} e^{-2 a_{s} \tau_{s}}-K^{T_{s} \bar{T}_{j}}\left(\partial_{\bar{T}_{j}} K\right) a_{s} A_{s} e^{-a_{s} T_{s}} \bar{W}-K^{T_{i} \bar{T}_{s}}\left(\partial_{T_{i}} K\right) a_{s} \bar{A}_{s} e^{-a_{s} \bar{T}_{s}} W\right] \\
& +\frac{3 \xi}{4 \mathcal{V}^{3}} e^{K(S, U)}|W|^{2}+\mathcal{O}\left(\frac{1}{\mathcal{V}^{4}}\right) \\
\simeq & e^{K(S, U)}\left[\frac{4}{\mathcal{V}} a_{s}^{2}\left|A_{s}\right|^{2}\left(-\kappa_{s s i} t^{i}\right) e^{-2 a_{s} \tau_{s}}-\frac{4}{\mathcal{V}^{2}} a_{s} \tau_{s}\left|A_{s} W\right| e^{-a_{s} \tau_{s}}+\frac{3 \xi}{4 \mathcal{V}^{3}}|W|^{2}\right]+\mathcal{O}\left(\frac{1}{\mathcal{V}^{4}}\right),
\end{aligned}
$$


in the large volume regime, where $D_{T_{i}} W=W_{T^{i}}+K_{T_{i}} W$ with $W_{T^{i}}=\partial_{T^{i}} W$ and the $\mathrm{CY}$ volume is taken as $\mathcal{V}=$ $\sum_{i j k} \frac{\kappa_{i j k}}{6} t^{i} t^{j} t^{k}$ with $\kappa_{i j k}$ being the intersection numbers among the two-cycles $t^{i}$ of CY. Now, the imaginary part of $T_{s}$ is set as its minimum and we use $K^{T_{i} \bar{T}_{j}} K_{\bar{T}_{j}}=-2 \tau_{i}+$ $\mathcal{O}\left(\mathcal{V}^{-1}\right)$ and $K^{T_{i} \bar{T}_{j}}=-4 \mathcal{V} \kappa_{i j k} t^{k}+4 \tau_{i} \tau_{j}+\mathcal{O}\left(\mathcal{V}^{-1}\right)$ [25], where the divisor volume is defined as $\tau_{i}=\frac{1}{2} \kappa_{i j k} t^{j} t^{k}$. Note that the first term in Eq. (55) is positive, since it is originating from the positive definite term. The simplified above scalar potential is a well-known form as discussed in the LVS, where the coefficient of $\alpha^{\prime}$-correction $\xi$ is positive, namely $h^{2,1}>h^{1,1}>1$. In LVS, the "small" modulus $\tau_{s}$ and volume modulus $\mathcal{V}$ are stabilized at

$$
\mathcal{V} \sim e^{a_{s} \tau_{s}}, \quad \tau_{s} \sim \xi^{2 / 3},
$$

because of the positivity of $\xi$.

On the contrary, in this paper, we proceed to discuss the opposite sign of $\xi$, namely $h^{1,1} \geq h^{2,1}>1$. The scalar potential is given by a sum of the $F$-term and leading radiative corrections,

$$
\begin{aligned}
V \simeq & e^{K(S, U)} M_{\mathrm{Pl}}^{4}\left[\frac{4}{\mathcal{V}} a_{s}^{2}\left|\hat{A}_{s}\right|^{2}\left(-\kappa_{s s i} t^{i}\right) e^{-2 a_{s} \tau_{s}}\right. \\
& \left.-\frac{4}{\mathcal{V}^{2}} a_{s} \tau_{s}\left|\hat{A}_{s} \hat{W}\right| e^{-a_{s} \tau_{s}}+\frac{3 \xi}{4 \mathcal{V}^{3}}|\hat{W}|^{2}+\frac{c_{1}}{32 \pi^{2}} \Lambda^{2} \frac{|\hat{W}|^{2}}{\mathcal{V}^{2} M_{\mathrm{Pl}}^{2}}\right] \\
& +\mathcal{O}\left(\frac{1}{\mathcal{V}^{4}}\right)
\end{aligned}
$$

where $A_{s}=\hat{A}_{s} M_{\mathrm{Pl}}^{3}$ and $W=\hat{W} M_{\mathrm{Pl}}^{3}$. In units of $\Lambda=$ $m_{\mathrm{KK}}=\sqrt{\pi} M_{\mathrm{Pl}} / \mathcal{V}^{2 / 3}=1$, the scalar potential reduces to

$$
\begin{aligned}
V \simeq & \frac{e^{K(S, U)}}{\pi^{2}}\left[4 \mathcal{V}^{5 / 3} a_{s}^{2}\left|\hat{A}_{s}\right|^{2}\left(-\kappa_{s s i} t^{i}\right) e^{-2 a_{s} \tau_{s}}\right. \\
& \left.-4 \mathcal{V}^{2 / 3} a_{s} \tau_{s}\left|\hat{A}_{s} \hat{W}\right| e^{-a_{s} \tau_{s}}+\frac{3 \xi}{4 \mathcal{V}^{1 / 3}}|\hat{W}|^{2}+\frac{c_{1}}{32 \pi} \frac{|\hat{W}|^{2}}{\mathcal{V}^{2 / 3}}\right] \\
& +\mathcal{O}\left(\frac{1}{\mathcal{V}^{4 / 3}}\right) .
\end{aligned}
$$

The extremal condition of $\tau_{s}$ reads

$$
\begin{aligned}
\frac{\partial V}{\partial \tau_{s}} \simeq & 4 \mathcal{V}^{5 / 3} a_{s}^{2}\left|\hat{A}_{s}\right|^{2} e^{-2 a_{s} \tau_{s}}\left[\frac{\partial t^{j}}{\partial \tau_{s}}\left(-\kappa_{s s j}\right)-2 a_{s}\left(-\kappa_{s s i} t^{i}\right)\right] \\
& -4 \mathcal{V}^{2 / 3} a_{s}\left|\hat{A}_{s} \hat{W}_{0}\right| e^{-a_{s} \tau_{s}}\left[1-a_{s} \tau_{s}\right] \\
\simeq & 4 \mathcal{V}^{5 / 3} a_{s} e^{-2 a_{s} \tau_{s}}\left[-2 a_{s}^{2}\left|\hat{A}_{s}\right|^{2}\left(-\kappa_{s s i} t^{i}\right)\right. \\
& \left.+\frac{e^{a_{s} \tau_{s}}}{\mathcal{V}}\left|\hat{A}_{s} \hat{W}_{0}\right| a_{s} \tau_{s}\right]=0,
\end{aligned}
$$

with $W_{0}=\hat{W}_{0} M_{\mathrm{Pl}}^{3}$, where we use $\partial t^{j} / \partial \tau_{s}<1$ and $a_{s} \tau_{s} \gg 1$ to suppress the higher instanton effects. In this way, we obtain

$$
\frac{e^{a_{s} \tau_{s}}}{\mathcal{V}}\left|\hat{A}_{s} \hat{W}_{0}\right| a_{s} \tau_{s}=2 a_{s}^{2}\left|\hat{A}_{s}\right|^{2}\left(-\kappa_{s s i} t^{i}\right)
$$

Another extremal condition of $\mathcal{V}$ gives rise to

$$
\begin{aligned}
\frac{\partial V}{\partial \mathcal{V}} \simeq & \frac{20 \mathcal{V}^{2 / 3}}{3} a_{s}^{2}\left|\hat{A}_{s}\right|^{2}\left(-\kappa_{s s i} t^{i}\right) e^{-2 a_{s} \tau_{s}}-\frac{8 a_{s} \tau_{s}}{3 \mathcal{V}^{1 / 3}}\left|\hat{A}_{s} \hat{W}_{0}\right| e^{-a_{s} \tau_{s}} \\
& -\frac{\xi}{4 \mathcal{V}^{4 / 3}}\left|\hat{W}_{0}\right|^{2}-\frac{c_{1}}{48 \pi} \frac{\left|\hat{W}_{0}\right|^{2}}{\mathcal{V}^{5 / 3}} \\
\simeq & \frac{2 a_{s} \tau_{s}}{3 \mathcal{V}^{1 / 3}}\left|\hat{A}_{s} \hat{W}_{0}\right| e^{-a_{s} \tau_{s}}-\frac{\xi}{4 \mathcal{V}^{4 / 3}}\left|\hat{W}_{0}\right|^{2}-\frac{c_{1}}{48 \pi} \frac{\left|\hat{W}_{0}\right|^{2}}{\mathcal{V}^{5 / 3}} \\
\simeq & \frac{1}{3 \mathcal{V}^{3 / 4}} \frac{\left(\tau_{s}\right)^{2}}{\left(-\kappa_{s s i} t^{i}\right)}\left|\hat{W}_{0}\right|^{2}-\frac{\xi}{4 \mathcal{V}^{4 / 3}}\left|\hat{W}_{0}\right|^{2}-\frac{c_{1}}{48 \pi} \frac{\left|\hat{W}_{0}\right|^{2}}{\mathcal{V}^{5 / 3}} \\
\equiv & -\frac{\hat{\xi}}{4 \mathcal{V}^{4 / 3}}\left|\hat{W}_{0}\right|^{2}-\frac{c_{1}}{48 \pi} \frac{\left|\hat{W}_{0}\right|^{2}}{\mathcal{V}^{5 / 3}},
\end{aligned}
$$

where

$$
\hat{\xi} \equiv \xi-\frac{4}{3} \frac{\left(\tau_{s}\right)^{2}}{\left(-\kappa_{s s i} t^{i}\right)} .
$$

Consequently, the volume modulus is stabilized at

$$
\mathcal{V} \simeq 18664\left(-\frac{c_{1} / \hat{\xi}}{10^{3}}\right)^{3},
$$

by replacing $\xi$ of Eq. (23) into $\hat{\xi}$. Although the $\hat{\xi}$ depends on the topology of CY manifold as in Eq. (23), the stabilization of volume modulus $\mathcal{V}$ and $\tau_{s}$ is achieved inside the Kähler cone with $\mathcal{V} \gg \tau_{s}>1$. Indeed, when $\kappa_{s s i} t^{i} \sim-\tau_{s}^{1 / 2}$, $\hat{A}_{s}=\hat{W}_{0}=1, \quad c_{1}=200, \quad \xi=-10^{-1} \quad$ and $\quad a_{s}=2 \pi$, Eqs. (60) and. (63) give

$$
\mathcal{V} \simeq 4098, \quad \tau_{s} \simeq 1.69 .
$$

Furthermore, even if the Euler number of CY is vanishing, i.e., $\xi=0$, both the moduli fields can be stabilized at $\mathcal{V} \simeq 3444, \tau_{s} \simeq 1.66$, where we set the same parameters of Eq. (64) except for $\xi$. Following the same procedure of Sec. III A, we can achieve the de Sitter vacuum by including the anti-D3 branes.

\section{E. Ultralight axion}

Finally, we take a closer look at the mass of axion associated with the overall volume modulus. To simplify our analysis, we take into account the $\mathrm{CY}$ volume dominated by the single Kähler modulus $T$, i.e., $\mathcal{V}=\kappa(T+\bar{T})^{3 / 2}$ with $\kappa$ being a positive real constant. When there exist the 
TABLE II. Axion mass in units of gravitino mass and its decay constant.

\begin{tabular}{lccccc}
\hline \hline Scale & $n=1$ & $n=3$ & $n=5$ & $n=7$ & $n=9$ \\
\hline$m_{\theta} / m_{3 / 2}$ & $2.3 \times 10^{-209}$ & $7.4 \times 10^{-68}$ & $9.7 \times 10^{-40}$ & $9.1 \times 10^{-28}$ & $3.7 \times 10^{-21}$ \\
$f_{\theta}[\mathrm{GeV}]$ & $3 \times 10^{15}$ & $9 \times 10^{15}$ & $1.5 \times 10^{16}$ & $2 \times 10^{16}$ & $2.7 \times 10^{16}$ \\
\hline \hline
\end{tabular}

D-brane instanton effects or gaugino condensation on D7-branes, the axion potential can be extracted from the superpotential

$$
W=W_{0}+A^{(T)} e^{-\frac{2 \pi}{n} T},
$$

where $W_{0}=\left\langle W_{\text {flux }}\right\rangle$ and $A^{(T)}$ are assumed to be real constants, and $n=1$ for the brane instanton and $n=N$ for the gaugino condensation on $N$ stacks of D7-branes wrapping the divisor with volume $\tau$. Such a nonperturbative superpotential term generates the axion potential,

$$
\begin{aligned}
V & =e^{K / M_{\mathrm{Pl}}^{2}} K^{T \bar{T}} K_{T}\left(\frac{W_{T} \bar{W}}{M_{\mathrm{Pl}}^{2}}+\frac{\bar{W}_{\bar{T}} W}{M_{\mathrm{Pl}}^{2}}\right) \\
& \simeq \frac{e^{K(S, U)} A^{(T)} W_{0}}{\mathcal{V}^{2} M_{\mathrm{Pl}}^{2}} \frac{(T+\bar{T})^{2}}{3} \frac{3}{T+\bar{T}} \frac{2 \pi}{n}\left(e^{-\frac{2 \pi}{n} T}+e^{-\frac{2 \pi}{n} \bar{T}}\right) \\
& \simeq \frac{e^{K(S, U)} A^{(T)} W_{0}}{\mathcal{V}^{2} M_{\mathrm{Pl}}^{2}}\left(\frac{\mathcal{V}}{\kappa}\right)^{2 / 3} \frac{4 \pi}{n} e^{-\frac{2 \pi}{n} \tau} \cos \left(\frac{2 \pi}{n} \sigma\right),
\end{aligned}
$$

where $T=\tau+i \sigma$. After canonically normalizing the axion $\theta=\sqrt{2 K_{T \bar{T}}} \sigma$, we obtain the tiny mass of axion and its decay constant,

$$
\begin{aligned}
m_{\theta} & \simeq m_{3 / 2} \frac{\left(\left|A^{(T)}\right| / M_{\mathrm{Pl}}^{3}\right)^{1 / 2}}{\sqrt{3} \kappa}\left(\frac{2 \pi}{n}\right)^{3 / 2}\left(\frac{\mathcal{V}}{\kappa}\right) e^{-\frac{\pi}{2 n}\left(\frac{\mathcal{V}}{\kappa}\right)^{2 / 3}}, \\
f_{\theta} & =\frac{n \sqrt{2 K_{T \bar{T}}}}{2 \pi}=\frac{\sqrt{6} n}{2 \pi}\left(\frac{\kappa}{\mathcal{V}}\right)^{2 / 3} M_{\mathrm{Pl}},
\end{aligned}
$$

where the $\mathrm{CY}$ volume and gravitino mass are given in Eqs. (39) and (43). Thus, large volume of CY results in the ultralight axion associated with the volume modulus in a way similar to the LVS.

Although the axion mass highly depends on the value of $n$, we list the typical axion mass in Table II setting the same parameters of Sec. II C and

$$
\kappa=1, \quad\left|A^{(T)}\right|=M_{\mathrm{Pl}}^{3}, \quad\left|c_{1} / \xi\right|=10^{3} .
$$

It is interesting to discuss the astrophysical and cosmological physics of such an ultralight axion, which will be studied in a separate work.

\section{CONCLUSION}

We have discussed the stabilization of Kähler moduli using the leading $\alpha^{\prime}$-corrections and the radiative corrections due to the sparticles within the framework of the type IIB superstring theory on Calabi-Yau orientifolds with D7-branes. When all the volumes of the divisors in CY threefold are sufficiently large in string units, the nonperturbative effects for the Kähler moduli are suppressed enough compared with $\alpha^{\prime}$-corrections and the radiative corrections. We find that these perturbative corrections give rise to the stabilization of the overall Kähler modulus for a general class of CY threefolds, only if the Euler number of $\mathrm{CY}$ is positive. Since the volume of CY scales as the number of sparticles, we require that the relatively large number of sparticles contributes to the Coleman-Weinberg potential through the radiative corrections to achieve the large CY volume. Such a large number of sparticle contributions could dominate over the string loop corrections to the Kähler potential discussed in Ref. [11] where one needs a certain amount of fine-tuning of the complex structure moduli to realize the large volume of $\mathrm{CY}$. Furthermore, our scenario of moduli stabilization does not require the tuning of flux-induced superpotential in contrast to the KKLT scenario. The Kaluza-Klein and stringy modes are sufficiently heavier than Kähler moduli and sparticles. In this reason, the low-energy effective action is controllable in the four-dimensional $N=1$ supergravity. The vacuum in our scenario of moduli stabilization is the ant-de Sitter minimum. The structure of moduli stabilization is still maintained even after we uplift the antide Sitter minimum to de Sitter vacuum by introducing antiD3 branes. Note that a requirement of the positive Euler number is different from the conventional large volume scenario, but CY threefolds with the positive Euler number are accounted for half of the CY threefolds in the sense of mirror symmetry.

However, the positive Euler number of CY threefold indicates that we have to take into account a lot of Kähler moduli compared with the complex structure moduli. We expect that the $D$-term contribution from fractional D3-branes and magnetized D7-branes would lead to the stabilization of these Kähler moduli except for the overall volume modulus as discussed in Sec. III C. The nonperturbative effects also allow us to stabilize some Kähler moduli without spoiling the stabilization of volume modulus as shown in Sec. III D. In this moduli stabilization scenario, the axion associated with the volume modulus remains light and it could be a target of astrophysical and cosmological observations. It is interesting to show explicit moduli stabilization in a detailed setup, but we leave it for a future work. 


\section{ACKNOWLEDGMENTS}

T. K. was is supported in part by MEXT KAKENHI Grant No. JP17H05395 and JSPS KAKENHI Grant No. JP26247042. H. O. was supported in part by JSPS KAKENHI Grant Number JP17K14303.

[1] K. Dasgupta, G. Rajesh, and S. Sethi, M theory, orientifolds and G-flux, J. High Energy Phys. 08 (1999) 023.

[2] Y. Honma and H. Otsuka, On the flux vacua in F-theory compactifications, Phys. Lett. B 774, 225 (2017).

[3] S. B. Giddings, S. Kachru, and J. Polchinski, Hierarchies from fluxes in string compactifications, Phys. Rev. D 66, 106006 (2002).

[4] S. Gukov, C. Vafa, and E. Witten, CFT's from Calabi-Yau four folds, Nucl. Phys. B584, 69 (2000); Erratum, Nucl. Phys. B608, 477 (2001).

[5] S. Kachru, R. Kallosh, A. D. Linde, and S. P. Trivedi, De Sitter vacua in string theory, Phys. Rev. D 68, 046005 (2003).

[6] V. Balasubramanian, P. Berglund, J. P. Conlon, and F. Quevedo, Systematics of moduli stabilisation in CalabiYau flux compactifications, J. High Energy Phys. 03 (2005) 007.

[7] O. Lebedev, H. P. Nilles, and M. Ratz, De Sitter vacua from matter superpotentials, Phys. Lett. B 636, 126 (2006).

[8] E. Dudas, C. Papineau, and S. Pokorski, Moduli stabilization and uplifting with dynamically generated F-terms, J. High Energy Phys. 02 (2007) 028; H. Abe, T. Higaki, T. Kobayashi, and Y. Omura, Moduli stabilization, F-term uplifting and soft supersymmetry breaking terms, Phys. Rev. D 75, 025019 (2007); R. Kallosh and A. D. Linde, O’kklt, J. High Energy Phys. 02 (2007) 002; H. Abe, T. Higaki, and T. Kobayashi, More about F-term uplifting, Phys. Rev. D 76, 105003 (2007).

[9] M. Cicoli, A. Maharana, F. Quevedo, and C. P. Burgess, De Sitter string vacua from dilaton-dependent non-perturbative effects, J. High Energy Phys. 06 (2012) 011.

[10] T. Kobayashi, N. Omoto, H. Otsuka, and T. H. Tatsuishi, Supersymmetry preserving and breaking degenerate vacua, and radiative moduli stabilization, Phys. Rev. D 96, 046004 (2017).

[11] M. Berg, M. Haack, and B. Kors, On Volume Stabilization by Quantum Corrections, Phys. Rev. Lett. 96, 021601 (2006).

[12] S. R. Coleman and E. J. Weinberg, Radiative corrections as the origin of spontaneous symmetry breaking, Phys. Rev. D 7, 1888 (1973).

[13] H. Jockers and J. Louis, D-terms and F-terms from D7brane fluxes, Nucl. Phys. B718, 203 (2005); R. Blumenhagen, S. Moster, and E. Plauschinn, Moduli stabilisation versus chirality for MSSM like type IIB orientifolds, J. High Energy Phys. 01 (2008) 058; J. P. Conlon, A. Maharana, and F. Quevedo, Towards realistic string vacua, J. High Energy Phys. 05 (2009) 109; H. Abe, T. Kobayashi, K. Sumita, and Y. Tatsuta, Supersymmetric models on magnetized orbifolds with flux-induced Fayet-Iliopoulos terms, Phys. Rev. D 95,
015005 (2017); H. Abe, T. Kobayashi, K. Sumita, and S. Uemura, Kahler moduli stabilization in semirealistic magnetized orbifold models, Phys. Rev. D 96, 026019 (2017).

[14] K. Becker, M. Becker, M. Haack, and J. Louis, Supersymmetry breaking and alpha-prime corrections to flux induced potentials, J. High Energy Phys. 06 (2002) 060.

[15] T. W. Grimm and J. Louis, The effective action of $N=1$ Calabi-Yau orientifolds, Nucl. Phys. B699, 387 (2004).

[16] V. S. Kaplunovsky and J. Louis, Model independent analysis of soft terms in effective supergravity and in string theory, Phys. Lett. B 306, 269 (1993).

[17] R. Barbieri and S. Cecotti, Radiative corrections to the effective potential in $N=1$ supergravity, Z. Phys. C 17, 183 (1983); M. Srednicki and S. Theisen, Supergravitational Radiative Corrections to the Gauge Hierarchy, Phys. Rev. Lett. 54, 278 (1985); G. A. Diamandis, J. R. Ellis, A. B. Lahanas, and D. V. Nanopoulos, Vanishing scalar masses in no scale supergravity, Phys. Lett. 173B, 303 (1986); M. Quiros, On the effective potential and gravitino mass determination in compactified superstring models, Phys. Lett. 173B, 265 (1986).

[18] M. Berg, M. Haack, and E. Pajer, Jumping through loops: On soft terms from large volume compactifications, J. High Energy Phys. 09 (2007) 031; M. Cicoli, J. P. Conlon, and F. Quevedo, Systematics of string loop corrections in type IIB Calabi-Yau flux compactifications, J. High Energy Phys. 01 (2008) 052.

[19] M. Cicoli, J. P. Conlon, and F. Quevedo, General analysis of LARGE volume scenarios with string loop moduli stabilisation, J. High Energy Phys. 10 (2008) 105.

[20] D. Ciupke, J. Louis, and A. Westphal, Higher-derivative supergravity and moduli stabilization, J. High Energy Phys. 10 (2015) 094.

[21] R. Minasian, T. G. Pugh, and R. Savelli, F-theory at order $\alpha^{\prime 3}$, J. High Energy Phys. 10 (2015) 050.

[22] L. E. Ibanez and A. M. Uranga, String Theory and Particle Physics: An Introduction to String Phenomenology (Cambridge University Press, Cambridge, England, 2012).

[23] R. Blumenhagen, B. Kors, D. Lust, and S. Stieberger, Fourdimensional string compactifications with D-branes, orientifolds and fluxes, Phys. Rep. 445, 1 (2007).

[24] M. Cicoli, D. Klevers, S. Krippendorf, C. Mayrhofer, F. Quevedo, and R. Valandro, Explicit de Sitter flux vacua for global string models with chiral matter, J. High Energy Phys. 05 (2014) 001.

[25] K. Bobkov, Volume stabilization via alpha-prime corrections in type IIB theory with fluxes, J. High Energy Phys. 05 (2005) 010; R. Altman, Y. H. He, V. Jejjala, and B. D. Nelson, New large volume solutions, arXiv:1706.09070. 